\title{
Genome-wide identification and characterization of lectin receptor-like kinase gene family in cucumber and expression profiling analysis under different treatments
}

Duo Lv

Shanghai Jiao Tong University

\section{Gang Wang}

Shanghai Jiao Tong University

Liang-Rong Xiong

Shanghai Jiao Tong University

Jing-Xian Sun

Shanghai Jiao Tong University

Yue Chen

Shanghai Jiao Tong University

Chun-Li Guo

Shanghai Jiao Tong University

Yao Yu

Shanghai Jiao Tong University

Huan-Le He

Shanghai Jiao Tong University

\section{Run Cai}

Shanghai Jiao Tong University

Junsong Pan ( $\boldsymbol{\sim}$ jspan71@sjtu.edu.cn )

Shanghai Jiao Tong University https://orcid.org/0000-0003-3983-1285

Research article

Keywords: Cucumber, Expression analysis, Genome-wide analysis, Lectin receptor-like kinase, Phylogenetic analysis

Posted Date: May 11th, 2020

DOI: https://doi.org/10.21203/rs.2.20023/v3 
License: (c) (i) This work is licensed under a Creative Commons Attribution 4.0 International License. Read Full License

Version of Record: A version of this preprint was published at Genes on September $2 \mathrm{nd}, 2020$. See the published version at https://doi.org/10.3390/genes11091032. 


\section{Abstract}

Background Lectin receptor-like kinases (LecRLKs) are a class of membrane proteins found in plants that are involved in diverse functions, including plant development and stress responses. Although LecRLK families have been identified in a variety of plants, a comprehensive analysis has not yet been undertaken in cucumber ( Cucumis sativus L.).

Results In this study, 46 putative LecRLK genes were identified in cucumber genome, including 23 G-type, 22 L-type and $1 \mathrm{C}$-type LecRLK genes. They unequally distributed on all 7 chromosomes with a clustering trendency. Most of the genes in the cucumber LecRLK ( Cs ecRLK) gene family lacked introns. In addition, there were many regulatory elements associated with phytohormone and stress on these genes' promoters. Transcriptome data demonstrated that distinct expression patterns of CsLecRLK genes in various tissues. Furthermore, we found that each member of the CsLecRLK family had its own unique expression pattern under hormone and stress treatment by the quantitative real time PCR (qRT-PCR) analysis.

Conclusion This study provides a better understanding of the evolution and function of LecRLK gene family in cucumber, and opens the possibility to explore the roles that LecRLK s might play in the life cycle of cucumber.

\section{Background}

Plants are immobile creatures compared with animals, so they have more sensitive signal sensors to cope with the changing environment around them. In the long-term evolutionary process, plants have evolved a complete set of signal receptor proteins. After receiving external stimulus, they would transmit signals to downstream pathways the first time to allow plant respond to the stimulus. Cell surface receptors, a kind of signal receptor proteins, play important roles in receiving and transmitting environmental signals. The receptor-like kinase (RLK) family, one important family of cell surface receptors, contains three kinds of domains, such as extracellular domain, transmembrane domain (TM) and intracellular kinase domain. RLK proteins could be classified into different families based on the structure of the extracellular domains and intracellular kinase domains.

Lectin receptor-like kinases (LecRLKs), a class of RLKs that contain a lectin domain within the extracellular domain, are a gene family that is specialized for sensing external environmental stimuli and transmitting signals. They are localized on the cell membrane, relying on $\mathrm{N}$ terminus diverse extracellular ligand recognition domains (also called lectin domain) to recognize various environmental stimulus, and then phosphorylate downstream proteins through their $\mathrm{C}$ terminus intracellular kinase domain to pass received signals (Bouwmeester and Govers, 2009).

Based on the identity of lectin domains, the LecRLKs have been divided into three subfamilies (Fig. 1): Ltype, G-type, and C-type LecRLKs (Vaid et al., 2012). These subclasses are very distinct from each other, with sugar-binding ability of lectin domain. The G-type LecRLKs possess a-D mannose specific plant 
lectins, which are also accompanied in most of the proteins (not necessarily all) by both Plasminogen Apple Nematode (EGF) domain and Epidermal Growth Factor (PAN) domain motif or one of them. The EGF motif is cysteine rich (Shiu, 2001) and probably takes part in the formation of disulfide bonds (Vaid et al., 2012). The PAN motif is believed to be involved in protein-protein and protein carbohydrate interactions (Naithani et al., 2007). As the name suggests the lectin domain of L-type LecRLKs resembles soluble lectin protein found in leguminous plants (Hervé et al., 1999). The third class of LecRLKs are Ctype lectin kinases. The C-type LecRLKs in plants are thought to be homologues of calcium-dependent lectin motifs which are a large group of mammalian proteins known to be involved in innate immune responses and pathogen recognition (Cambi et al., 2005). Though C-type LecRLKs are present in large number in mammalian system, only one gene encoding for C-type LecRLK exists in rice and Arabidopsis respectively (Bouwmeester and Govers, 2009).

The roles of $L e c R L K$ s in plants are believed to be primarily involved in plant development, innate immunity and abiotic responses. Previous reports have confirmed that LecRLK family is involved in plant root development (Cheng et al., 2013), pollen development (Wan et al., 2008), cotton fiber development (Zuo et al., 2004), and hormone signal recognition (Deng et al., 2009). At the same time, LecRLKs also play an irreplaceable role in plant resistance to diseases, insect pests and stresses, such as salt stress ( $\mathrm{Li}$ et al., 2014; He et al., 2004), wounding (Riou et al, 2002), and fungal pathogen (Ohtake, 2000; Desclos et al., 2012). Although there is only one subfamily member in Arabidopsis and rice, the C-type LecRLKs had not been reported involving any specific biological traits for a long time. Until 2017, a C-type LecRLK was identified that may affect morphogenesis of trichome in cucumber (Cucumis sativus) (Guo et al., 2017).

Genome-wide analysis of the LecRLK gene family has been reported in some plants (Vaid et al., 2012; Shumayla et al., 2016; Yang et al., 2016; Liu et al., 2018) , but there are only a few early studies reporting on the presence of LecRLKs (Wu et al., 2014). A comprehensive understanding of LecRLKs in cucumber is still lacking. Here, we did a complete identification and analysis of the entire LecRLK gene family in cucumber, 46 LecRLK genes (CsLecRLKS) were identified in cucumber genome. Furthermore, we analyzed their phylogenetic relationship, gene structure, conserved domain, gene duplications, chromosome distribution and cis-acting elements on the promoters. Finally, we profiled the expression of the predicted

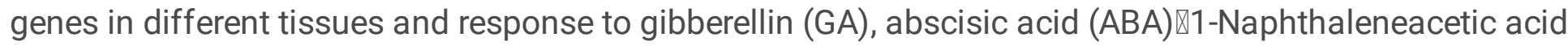
(NAA), indole-3-acetic acid (IAA) and cold treatments in cucumber. Our study provides valuable information for further functional research on the LecRLK gene family in cucumber.

\section{Results}

\section{Genome-wide identification of the LecRLKs in cucumber}

We identified a total of 46 LecRLKgenes which named CsLecRLKs in the cucumber genome by the Pfam and SMART search, their sequences of CDS and protein were listed in Table S4-S9. The total number of LecRLKs in cucumber is less than that in Arabidopsis (75 LecRLK genes) or rice (173 LecRLK genes) (Vaid et al., 2012). The 46 CsLecRLKs were classified into 23 G-type, 22 L-type, and one C-type based on 
their extracellular lectin domain. The molecular weight (MW) of the proteins ranged from $62.5 \mathrm{kDa}$ (Csa1G056960) to $94.5 \mathrm{kDa}$ (Csa3G733860), and the isoelectric point (Ip) ranged from 4.98 (Csa4G289630) to 9.51 (Csa1G056960), the range of CDS length was 1,803-2,502bp. With the predicted protein structures, it could be considered that most of the CsLecRLKs were localized on the plasma membrane, only Csa7G045520 was located on the extracellular. More information of CsLecRLKs, including the length of the gene, the length of CDS, the length of the protein sequence, the protein MW and $\mathrm{pl}$ were listed in Table 1.

By analyzing the molecular weight of all 46 CsLecRLKs, we found that the weight of G-type CsLecRLKs $(83.2 \mathrm{kDa})$ are generally larger than L-type $(62.5 \mathrm{kDa})$ and C-type $(74.6 \mathrm{kDa})$. This may be mainly due to the fact that in addition to the lectin domain, G-type CsLecRLKs often contain the EGF and PAN domains (Fig. 1). Signal peptides and transmembrane domain (TM) are critical for protein localization. The software prediction indicated that not each CsLecRLK had signal peptide and unique TM domain. The loss of signal peptide or TM domain would directly affect the localization of proteins in cells (Table 1). The plasma membrane localization of most of the CsLecRLKs indicated that they are signal receptors which can sense extracellular signals and then transmit the signals to the interior of the cells.

\section{Phylogenetic analysis of the CsLecRLKs}

We constructed an unrooted phylogenetic tree by the MEGA 7.0.21 (Fig. 2). As expected, the phylogenetic tree showed that the CsLecRLK family could be classified into three subgroups of L-type, G-type, and Ctype. This result is consistent with the domain-based classification of CsLecRLK family. The phylogenetic tree indicated that the L-type and C-type had a closer relationship. This result was different from previous reports in Arabidopsis and rice, which revealed a closer genetic relationship between G-type and L-type (Vail et al., 2012). As shown in Fig. 3, the phylogram of G-type and L-type CsLecRLKs could be divided into four and three sub-groups respectively. The division of individual clades was supported by high bootstrap values.

\section{Exon-Intron Structural Analysis of CsLecRLKs}

The genomic sequence and corresponding cDNA sequence of the CsLecRLKs were submitted to GSDS (Gene Structure Display Server) together for analyzing their gene structure (Fig. 3). The genome sequence lengths of CsLecRLKs ranged from 1803bp to $6481 \mathrm{bp}$, the lengths of CDS ranged from $1674 \mathrm{bp}$ to 2502 bp. The number of exon of these genes varied from one to nine, $80 \%$ CsLecRLKs had less than three exons. Excepted that Cs4G296230 contains three exons, all L-type CsLecRLKs contained only one or two exons, and the C-type CsLecRLK (Csa1G056960) contains four exons. The G-type CsLecRLKs contain one to nine exons. Among them, the Csa7G446780 contains nine exons, which has the most exons.

\section{Protein domain and Motif analysis of CsLecRLKs}

Through the SMART program prediction, we investigated conserved domains that present in CsLecRLKs. C-type and L-type CsLecRLKs were both only contain three based categories domain, Lectin domain, 
Transmembrane domain and Kinase domain. But some G-type CsLecRLKs also contained other two categories domains, PAN domain and EGF domain. Among G-type CsLecRLKs, ten proteins contain PAN and EGF domains at the same time, five proteins only contain PAN domain, eight proteins only contain EGF domain, and only one contains neither PAN domain nor EGF domain. Our result indicated that signal peptide would be not necessary to CsLecRLKs. There are 25 CsLecRLKs without signal peptide and 8 CsLecRLKs with more than two transmembrane domains.

Ten conserved motifs were identified in CsLecRLKs using the MEME program (Fig. 3). These motifs were labelled Motif 1 to Motif 10 from the $\mathrm{N}$ - to the $\mathrm{C}$-terminus. The details of the conserved motifs were shown in Fig. S1. The lengths of these motifs ranged from 15 to 60 residues. Generally, the CsLecRLKs contains 4 to 10 motifs. None of the motifs appeared in all gene family members. Excepted that Motif 8 and Motif 9 were only present in the G-type CsLecRLKs, other motifs were present in three type CsLecRLKs. With the CDD program (https://www.ncbi.nlm.nih.gov/cdd/), we found that the six of these motifs represent different kinase domains (Fig. S1), indicating that there may be multiple phosphorylation catalytic sites in each of CsLecRLKs.

\section{Chromosomal Location and Gene Duplication of CsLecRLKs}

We extracted the location data of CsLecRLKs and the length data of each chromosome from the cucumber genome annotation files by a series of Perl scripts, and constructed gene location map using MapChart software. As shown in Fig. 4, all CsLecRLKs were unevenly distributed across 7 cucumber chromosomes, and genes from the same subfamily on the same chromosome had a tendency to cluster. The number of CSLecRLKs on each chromosome varied from 1 to 7, chromosome 3 contains the largest number of 12 CsLecRLKs and chromosome 2 had only two CsLecRLKs.

During the biological evolution, the generation of gene family could be caused by tandem duplication and segmental duplication (Kent et al., 2003; Mehan et al., 2004). In order to explore whether CsLecRLK gene family also have an expansion caused by the two kinds of duplication, we analyzed the duplication events of CsLecRLK genes. The result indicated that although many genes were clustered on the chromosomes, only Csa1G071170 and Csa1G071160 were a pair of tandem duplicated genes, their divergence time was about 38.606 million of years ago (MYA). The other two pairs of duplicated events, Csa1G073890 and Csa7G048050, and Csa3G734030 and Csa4G296230, may be caused by duplication or ectopia of chromosome fragments during the evolution. These duplicated genes are not in the same chromosome. Their divergence times were 30.96 and $32.35 \mathrm{MYA}$, respectively. Based on the above results, it could be inferred that tandem duplication contributed to the expansion of CsLecRLKgene family.

\section{Cis-acting Elements Analysis on CsLecRLKs promoter}

Different genes have their own specific or consensus cis-acting elements on their promoters. Trans-acting factors bind to the cis-acting elements to regulate the gene expression. Different cis-acting elements may correspond to different biotic or abiotic stress signals which could induce or inhibit the genes expression. 
Therefore, the cis-acting elements analysis on CsLecRLKs promoter will help us to further understand these genes' function. We used Plantcare website to analyze the promoters of $1500 \mathrm{bp}$ upstream sequence from translation initiation site of CsLecRLKs, and found that there were 54 typical and functional cis-acting elements (Fig. S2), which could be divided into four types: light response, stress resistance, plant hormone and others. Among them, 24 cis-acting elements were related to light response, 11 were related to hormone included salicylic acid (SA), jasmonic acid (JA), ethylene, gibberellin and auxin, and 9 were abiotic stress elements. These results suggested that the CsLecRLK gene family may be mainly involved in the biological pathway of stress resistance in cucumber. There were six developmentally related cis-acting elements, five of which were related to seed development, suggesting that this gene family may play a role in seed development. More details were shown in the Table S1.

\section{Expression Pattern Analysis of CsLecRLK genes}

Little is known about the functions of LecRLKs in cucumber. As a first attempt to provide insights into their potential functions, we used RNA-Seq data from 10 tissues of cucumber to investigate the expression of each CsLecRLK gene (Table S2). Most of CsLecRLKs were expressed at a low level, some (Csa6G338050, Csa1G071160, and Csa3G115090) were barely expressed in any tissue.

Most of CsLecRLKs were barely expressed in male flower and unfertilized ovary (Fig. 5A), just 21 genes had a constitutive expression pattern (FPKM >= 1 in all tissues, Tao et al., 2018) in each of them. But in other tissues, each contains at least 25 constitutive expression pattern genes.

The expression pattern of all CSLeCRLKs could be divided into 3 groups based on their expression level in each tissue (Fig. 5A). From group 1 to 3 , the range and level of gene expression decreased successively. Group 1 contained 7 genes, which had a high expression level in each tissue with average FPKM of 18.01 . There were 12 genes belonged to Group 2, they had an intermediate expression level in each tissue with average FPKM of 6.48. Group 3 included 27 genes expressing at low level in each tissue with average FPKM of 1.29. Excepted that C-type CsLecRLK(Csa1G056960) belonged to group 1, and that L-type CsLecRLKs had higher expression level than G-type.

Thirty-six CsLecRLKs were expressed in all tissues (FPKM $>0$ in all tissues) (Tao et al., 2018) and 17 genes were constitutively expressed (FPKM $>=1$ in all tissues). Then we focused on those genes with relatively high expression (FPKM >=2 in all tissues) (Tao et al., 2018) and selected 5 tissues of cucumbers for cluster analysis (Fig. 5B), including root, hypocotyl, cotyledon, true leaf and tendril. We found a total of 16 genes were expressed in all these tissues. Specially, two genes were expressed only in the roots (Csa2G439210 and Csa3G115060), one gene (Csa3G099580) was expressed in tendril, and two genes just expressed in cotyledon (Csa7G446780 and Csa7G067410).

\section{Expression analysis of $C s L e c R L K$ genes in response to different treatments}

Gene expression is not only spatiotemporal specific but also can be induced or repressed by hormones and stress. Because most of LecRLKs are receptor proteins on the membrane, they usually can sense 
those stimuli at the first time and send signals to intracellular receptors. To uncover all the divergence information of CsLecRLKs under different environment for a short time, the expression patterns under different hormone treatments, including IAA, GA, ABA, and NAA, and cold stress treatments, were analyzed by qRT-PCR. The result showed that most of CsLecRLKs (31/46) responded to at least one treatment (Fold change $>1$ than the control group Significance $p=0.05$ ). Overall, there were 20 upregulated events and 38 downregulated events totally (Significance $p=0.05$ ). In order to show the experimental results more conveniently and intuitively, the change fold under different treatment was displayed in heatmap (Fig. 6) based on the data of qRT-PCR. Firstly, some CsLecRLKs (7/46) could be induced or repressed by multiple treatments (treatment number $>3$ ), for instance, Csa1G071170 could be induced by GA, IAA, NAA and ABA treatments, Csa4G005510 was repressed by all treatments except ABA. Secondly, the expression of different CsLecRLKs could be induced by different treatments. The cold stress induced or repressed the minimal CsLecRLKs gene expression, there were 4 genes expression had changed, they were downregulated. On the contrary, NAA induced or repressed the most CsLecRLKs gene expression, there were 20 genes that responded to NAA treatment, 6 genes were upregulated and 14 genes were downregulated. The 16 CsLecRLKs changed their expression level under ABA treatment, there were 8 genes upregulated and 8 genes downregulated. IAA and GA caused expression level change in 9 and 8 genes respectively. The IAA treatment caused 1 genes expression upregulated, and 8 genes downregulated. The GA treatment caused 5 gene expression upregulated and 3 downregulated. Thirdly, 14 CsLecRLKs had different expression pattern under various treatments, for example, Csa3G734030 could be induced by NAA, and repressed by ABA, Csa1G071150 was upregulated under ABA treatment, and downregulated under NAA and IAA treatments. The results indicated that the members of CsLecRLKs had their own response characteristics to hormones and stresses and may play an important role in sensing external stimulus signals. For example, although Csa1G071160 and Csa3G115090 were not expressed in the root, our experiment showed that they can be induced by NAA and ABA, respectively. There were 15 genes did not have significance expression change under different treatment, they were Csa1G056960, Csa7G029930, Csa5G550210, Csa4G296250, Csa7G048050, Csa1G073890, Csa4G289620, Csa3G115060, Csa3G099580, Csa1G071270, Csa6G516770, Csa2G439150, Csa1G605730, Csa6G338050 and Csa5G648630.

\section{Discussion}

In this work, we identified 46 LecRLK genes in cucumber genome, Based on the analysis of phylogenetic relationship, they were classified into three group, this result was consistent with the $\mathrm{N}$-terminal domain differences of the three subfamilies. All 46 LecRLK genes were distributed on seven chromosomes of the cucumber with a cluster tendency. Compared with other plants, cucumber contains fewer members of LecRLK gene family, there are only 46 CsLecRLKs in cucumber genome, among which 23 G-type, 22 Ltype and only one C-type. The number of $L e c R L K$ family members varies from different plants, this may be caused by the following three reasons. Firstly, the genome sizes of various plants are different, for example, the genome size of soybean is above $1 \mathrm{~GB}$, which contains 52,051 protein-coding genes (Shen et al., 2018), so soybean has more members of LecRLK than other plants (soybean contains 189 LecRLK 
genes) (Liu et al., 2018). Secondly, different numbers of LecRLKs are also related to the function of LecRLKs in different plants' life activities, for instance, G-type LecRLKs are generally considered as a class of proteins that may be involved in self-incompatibility because some G-type LecRLKs contain a Sdomain (Bouwmeester and Govers, 2009), which is an essential domain for sporophytic self-

incompatibility response-related proteins. The cucumber is a kind of unisexual flower plant that does not have the problem of identifying the source of pollen during reproduction, unlike Populus and Eucalyptus, which are obligate outcrossing plant, this may be partially explain that why Populus and Eucalyptus contain more G-types LecRLKs, leading to a big increase in the number of members of the entire gene family (Yang et al., 2016). Thirdly, there are fewer duplicated events in CsLecRLK gene family. Previous research has revealed three Whole-genome duplication (WGD) events in Arabidopsis (Cannon et al., 2004). However, cucumber lacks recent WGD events (Huang et al., 2009), we only identified three pairs of duplicate genes in cucumber. Although the genome of cucumber (genome size of $367 \mathrm{Mb}$ ) is larger than the genome of Arabidopsis (genome size of $125 \mathrm{Mb}$ ) (Huang et al., 2009), cucumber still has less LecRLKs (contains 46 LecRLK genes) than Arabidopsis (contains 75 LecRLK genes).

We identified 10 motifs exist in 46 CsLecRLKs, six of these motifs were located at the C-terminal of CsLecRLKs and were related to the catalytic activity of kinases. This result suggested that although the whole gene family was divided into three subfamilies according to the N-terminal lectin domain, members of the same subfamily also had different catalytic targets. It greatly enhanced the functional diversity of the CsLecRLKs, allowing the whole family to participate in more biological pathways.

Another interesting finding in our study was that most CsLecRLK genes have less introns, it is similar to previous studies in other plants. For example, most members of LecRLK gene family in soybean only have one or even no intron (Liu et al., 2018). Previous study in Arabidopsis and rice also indicated that there are few genes with introns in this gene family, containing only five and eight in Arabidopsis (contains 75 LecRLK genes) and rice (contains 173 LecRLK genes) respectively (Vaid et al., 2012). The reason for this kind of structure is probably that because the LecRLKs act as the signal receptors in plant, less introns means less selective cutting and splicing, this could save more transcription time, so that the signal could be transmitted at first time (Jeffares et al., 2008).

The LecRLK is a special family in plant genome, to date, no homologs of LecRLKs have been reported in the genomes of fungus and human (Yang et al., 2016). As the singal station of the immovable creatures, unique characteristic of $L e c R L K$ family may be closely related to their function on sensing the external environment. Some LecRLKs have been reported that involved in sensing invasion of microorganisms. The SD1-29 is one kind of G-type LecRLK in Arabidopsis, it can identify lipopolysaccharide which is a secretion from Gramnegative Pseudomonas and Xanthomonas (Ranf et al., 2015). Some LecRLKs will change their expression pattern with the changes of hormone and nutritional conditions. For example, the SIT1, a L-type LecRLK in rice, can mediate salt sensitivity. With the increase of $\mathrm{NaCl}$ concentration, the SIT1 was activated rapidly, which reduced the survival of rice (Li et al., 2014). In addition, some LecRLKs affect the development and growth of plant, for instance, two L-type LecRLKs, LecRK-IX.1 and LecRK-IX.2 will induce cell death, thereby increasing plant survival, when infected with phytophthora (Wang et al., 
2015). A C-type LecRLK mutant changed cell stacking pattern of trichome in cucumber, resulting fruit spines being easy to fall off from pericarp (Guo et al., 2017). It is similar to previous study (Yang et al., 2016), through the RNA-Seq data, we also found drastic number of CsLecRLKs may be expression in root (Fig. 6), this may be because root is one of the most important organ of plant, its role in fixation, temperature sensing, and nutrient absorption is irreplaceable. Tendrils serve as tentacles and are used to help cucumber climb. Cotyledon is the first tissues to feel the light when cucumber out of soil. Both of them are very important in feeling the environmental signals around cucumber. In the RNA-Seq data (Fig. 5), tendril and cotyledon were shown have higher CsLecRLKs expression level than other tissues, they both have 36 CsLecRLKs were constitutively expressed. Those results suggest that the CsLecRLK family does play a crucial role in sensing environmental signals. The result of promoter analysis shown that the number of light related cis-acting elements is the largest. Cotyledon has the most number of CsLecRLKs which have constitutively expressed. This indicates that CsLecRLKs play a role in sensing the light signal when plant out of the soil. We also found a number of CsLecRLKs whose expression levels were induced or repressed through hormone and stress treatment, implying that the expression level of these CsLecRLKs may be affected by biotic or abiotic stimuli not contained in our tested tissues. Of course, there are also some $C s L e c R L K s$ whose expression levels were not only low in tested tissues, but also not induced or repressed in our hormones and stress treatments, this may be because the stimulation conditions that relate to these genes were not found in our experiment, such as salt stress, insect stress, salicylic acid, ethylene and so on, these stimulation condition represents an exploratory area for further investigation.

\section{Conclusions}

In this study, a total of 46 CsLecRLKs were identified by bioinformatics methods, most of them lacked introns. 46 CsLecRLKs were clustered phylogenetically into three distinct subfamilies, C-type CsLecRLK and L-type CsLecRLK had a closer relationship. Through the ciselements analysis, many regulatory elements associated with phytohormone and stress were found on CsLecRLKs' promoters. Transcriptome data demonstrated that distinct expression patterns of CsLecRLK genes in various tissues. In different tissues, most of CsLecRLKs were expressed at a low level, three CsLecRLKs were even barely expressed in any tissue. In addition, the quantitative real time PCR (qRT-PCR) analysis showed that each member of the CsLecRLK gene family had its own unique expression pattern under hormone and stress treatment. The information obtained in the current study could help to provide a clear understanding of the feature of $L e c R L K$ gene family in cucumber.

\section{Methods}

\section{Identification of the LecRLK genes in cucumber}

The whole genome and protein sequence data of cucumber were downloaded from a public database (http://cucurbitgenomics.org/) (Cucumber (Chinese Long) genome v2). The Hidden Markov Model (HMM) was used to identify cucumber LecRLK candidates, and the HMM profiles of LecRLKs were 
downloaded from the Pfam protein database (http://pfam.xfam.org/), the models for these files are Ltype (Lectin_legB PF00139), G-type (B_lectin PF01453) and C-type (Lectin_C PF00059). We used HMMER 3.0 (Johnson et al., 2010) to search three types of genes from cucumber protein sequence data with e-

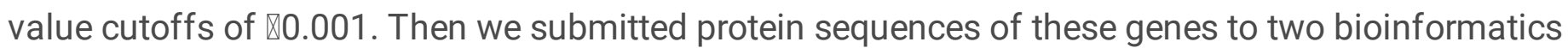
websites, Pfam (Finn et al., 2016) (http://pfam.xfam.org/) and SMART (Letunic et al., 2017) (http://smart.embl.de/), with e-value cut-off 1.0, and retaining those genes that contains lectin domain, transmembrane domains and kinase domains at the same time. The LecRLK family data of rice and Arabidopsis were downloaded from a previous research literature (Vaid et al., 2012).

\section{Phylogenetic analysis}

The full-length protein sequences of CsLecRLKs were aligned by the MUSCLE program with the default parameters (Edgar, 2004). The phylogenetic tree was constructed through Neighbor-Joining (NJ) method by MEGA 7.0.21, with the following parameters: Poisson model, pairwise deletion, and 1000 bootstrap replications.

\section{Conserved domain, motif identification and gene structure analysis}

The conserved motifs of the CsLecRLKs were predicted by the MEME program (Bailey, 1994) (http://meme-suite.org/), the parameters were set as any number of repetitions, optimum motif width of 6-210 residues, and searching for 10 motifs, site distribution mode was zoops, the maximal search time was 18000s. The CDD program (https://www.ncbi.nlm.nih.gov/cdd/) was used to predict the function of each conservative motif with e-value threshold 0.01. The Gene Structure Display Server (Hu et al., 2014) (http://gsds.cbi.pku.edu.cn/) was used to show the exon-intron structures of CsLecRLK genes. The Adobe Illustrator CS6 was used to beautify figures.

\section{Gene location and duplication analysis of CsLecRLKs}

A series of in-house Perl scripts was used to retrieve the location information of each CsLecRLK and the length of each cucumber chromosome from a whole genome sequence data of cucumber, which was downloaded from a public database (http://cucurbitgenomics.org/) (Cucumber (Chinese Long) genome v2). Then gene location information and length of each chromosome were displayed as visualized pictures through MapChart (Voorrips, 2002). The Adobe Illustrator CS6 was used to beautify the picture.

We used two methods to find duplication events among the CsLecRLKs. One way was that Gene duplication was confirmed with three criteria by an in-house Perl scripts: (a) the shorter aligned gene covered $>70 \%$ of the longer gene in length; (b) the similarity of aligned sequences was $>70 \%$ (Gu, 2002; Yang, 2008). (c) Two genes located in the same chromosomal fragment of less than $100 \mathrm{~kb}$ and separated by five or fewer genes were identified as tandem duplicated genes (Mehan et al., 2004). Another way was that we used the Multiple Collinearity Scan toolkit (MCScanX) to analyze the gene duplication events, with the default parameters (Wang et al., 2012). 
Ks (synonymous substitution rate) and Ka (nonsynonymous substitution rate) values of tandem duplicated genes were calculated by the method of Nei and Gojobori as implemented in KaKs_calculator (Zhang et al., 2006) based on the coding sequence alignments. The divergence time was calculated based on the formula $T=K s / 2 r$, with $K s$ being the synonymous substitutions per site and $r$ being the rate of divergence for nuclear genes from plants. The $r$ was taken to be $1.5 \times 10^{-8}$ synonymous substitutions per site per year for dicotyledonous plants (Olds et al., 2000).

\section{Analysis of cis-acting elements}

The upstream 1500bp of each CsLecRLK was obtained from the genome annotation files of Cucurbit Genomics Databases (http://cucurbitgenomics.org/) by a series of in-house Perl scripts, then scanned cis-acting elements contained in these sequences using the Plantcare Databases (http://bioinformatics.psb.ugent.be/webtools/plantcare/html/), in the process of analysis, we filtered out the cis-acting elements that are ubiquitous in most genes, for example CAAT-box, TATA-box, TATC-box and so on, only showing that may be typical and functional cis-acting elements presented in the CsLecRLK gene family. Then, the structures and annotations of the promoters were generated by GSDS (http:// gsds.cbi.pku.edu.cn/;) (Hu et al., 2015).

\section{Expression pattern analysis}

To analyze the expression profiles of cucumber $C s L e c R L K$ genes in different organs $₫$ we retrieved public RNA-Seq data (accession number: SRP071224) from the National Center for Biotechnology Information Short Read Archive database. Analysis of RNA-Seq data from 10 sampled cucumber tissues was performed based on the regular protocol by Wei et al (2016). These tissues of cucumber include root (4week-old seedlings), hypocotyl (4-week-old seedlings), cotyledon (4-week-old seedlings), true leaf (4-weekold seedlings), stem (12-week-old cucumber plants), tendril (12-week-old cucumber plants), female flower (12-week-old cucumber plants), male flower (12-week-old cucumber plants), ovary (unfertilized, 12-weekold cucumber plants), peel (unfertilized ovary, 12-week-old cucumber plants). The Heatmap of the gene Log2 (FPKM+1) values in 10 tissues of CsLecRLKs was drawn by the R program (3.5.2). To show which genes are widely expressed in different tissues, we also selected 5 typical tissues, included root (4-weekold seedlings), hypocotyl (4-week-old seedlings), cotyledon (4-week-old seedlings), true leaf of (4-week-old seedlings) and tendril, to draw Venn diagrams using the R program (3.5.2).

\section{Hormones and cold treatments}

The typical cucumber line '9930' was used as the experimental material to investigate the expression pattern in response to various phytohormones and cold treatments. Cucumber seeds were soaked in $55^{\circ} \mathrm{C}$ water for $2 \mathrm{~h}$ and germinated on petri dish in a growth chamber at $28^{\circ} \mathrm{C}$ in the dark for $2 \mathrm{~d}$. The germinated seeds were grown in pots containing peat: vermiculite mixture (3:1) in the greenhouse of Shanghai Jiao Tong University, and the controlled environment growth chamber was programed for light $16 \mathrm{~h} / 25^{\circ} \mathrm{C}$ and dark $8 \mathrm{~h} / 20^{\circ} \mathrm{C}$. After germination for 4 weeks, the seedlings were placed into hydroponic boxes with $1 / 2 \mathrm{MS}$ liquid solution (pH 5.8, without sugar) for 1 week to adapt to the environment (root 
was lucifugal), and then were treated with $100 \mathrm{mM}$ indole-3-acetic acid (IAA), $100 \mathrm{mM} \mathrm{1-}$ Naphthaleneacetic acid (NAA), $100 \mathrm{mM}$ abscisic acid (ABA), or $100 \mathrm{mM}$ gibberellin (GA) for $3 \mathrm{~h}$ under the same growth conditions as described earlier, respectively. The 1/2 MS liquid solution without any hormones was used as a control. Another group of seedlings was treated at $4^{\circ} \mathrm{C}$ for $1 \mathrm{~h}, 25^{\circ} \mathrm{C}$ was used as a control. Each treatment consisted of three biological replicates.

\section{RNA extraction and gene expression analysis}

Roots were collected from plants treated with different hormones, and young leaves (the second node from the top) were collected from plants subject to $4^{\circ} \mathrm{C}$. Total RNA was extracted using the RNeasy Plant Mini Kit (Cwbio, Beijing, China). The first strand cDNA was prepared according to the PrimeScript RT reagent Kit with gDNA Eraser (Cwbio, Beijing, China) protocol. To identify the relative expression level of different LecRLK genes under different treatment, qRT-PCR was conducted using FastStart Essential DNA Green Master (Roche, Mannheim, Germany). CsActin3 (Csa6G484600.1) was used as an internal control. qRT-PCR was performed in a total volume of $20 \mu \mathrm{L}$, containing $2 \mu \mathrm{L}$ of cDNA, $10 \mu \mathrm{L}$ SYBR mix, $2 \mu \mathrm{L}$ genespecific primers $(10 \mu \mathrm{M})$ and $6 \mu \mathrm{ddH}_{2} \mathrm{O}$, using the CFX Connection Real-Time System (Bio-Rad,

California, USA) with 40 cycles of $5 \mathrm{~s}$ at $95^{\circ} \mathrm{C}, 30 \mathrm{~s}$ at $60^{\circ} \mathrm{C}$. Each experiment was repeated three times, and each experiment included three biological repeats. Geneious software (version 2019.0.3) was used to design primers according to the cDNA sequences (Table S3). PCR amplified product lengths were about $150 \mathrm{bp}$. The data from real-time PCR amplification was analyzed using $2^{-\triangle \triangle C T}$ method at first (Livak and Schmittgen, 2000). Then we divided the result of each gene under different treatments by the result of the control group, and got fold change. Statistical difference was determined by t-test $\left({ }^{*} P<0.05\right)$ using Microsoft Excel 2010. The Heatmap of the change fold values of CsLecRLKs under five treatments was drawn by the $\mathrm{R}$ program (3.5.2).

\section{Abbreviations}

LecRLKs: Lectin receptor-like kinases; CsLecRLK: cucumber Lectin receptor-like kinases; qRT-PCR: quantitative real time PCR; RLK: receptor-like kinase; TM: transmembrane domain; MW: Molecular weight; Ip: Isoelectric point; GA: gibberellin, ABA: abscisic acid, NAA: 1-Naphthaleneacetic acid, IAA: indole-3acetic acid; MYA: Millions of years ago; SA: salicylic acid, JA: jasmonic acid; FPKM: Fragments per Kilobase Million, WGD: Whole genome duplication, PAN domain: Plasminogen Apple Nematode domain, EGF: Epidermal Growth Factor domain.

\section{Declarations}

\section{Ethics approval and consent to participate}

Not applicable, as this study did not involve human or animal subjects, and the seeds of cucumber were stored in School of Agriculture and Biology, Shanghai Jiao Tong University.The experiments comply with the current laws of the country in which we were performed. 


\section{Availability of data and materials}

The Illumina RNA-Seq data generated from 10 sampled cucumber tissues are available in the NCBI SRA with accessions number SRP071224. All data generated or analysed during this study are included in this published article and its supplementary information files.

\section{Consent for publication}

Not applicable.

\section{Competing interests}

The authors declare that they have no conflict of interest.

\section{Funding}

This study was supported by the National Natural Science Foundation of China (No. 31672148), it supported us in RNA sequencing, experiment of hormones and qRT-PCR.

This research was supported by the Foundation of Shanghai Municipal Commission of Science and Technology (Grant No.18391900300), this foundation supported us in data analysis and essay writing.

\section{Authors' contributions}

DL contributed to bioinformatics analysis and writing of the manuscript. LRX and YY helped with the RNA extraction and qRT-PCR. HLH, JXS and CLG helped with growing plants and experiment of hormones. YC contributed to data of RNA-Seq. RC, GW and JSP provided critical insights and revised the manuscript. All authors read and approved the final manuscript.

\section{Acknowledgements}

We thank Ling Dai (School of Electronic Information and Electrical Engineering, Shanghai Jiao Tong University, China) for helping with bioinformatics analysis, and Wencan Zhang for revising the 
manuscript (School of Naval Architecture, Ocean \& Civil Engineering, Shanghai Jiao Tong University, China).

\section{References}

Bailey T L, Nadya W, Chris M, et al. MEME: discovering and analyzing DNA and protein sequence motifs[J]. Nucleic Acids Research (suppl_2):suppl_2.

Bouwmeester K, Govers F. Arabidopsis L-type lectin receptor kinases: phylogeny, classification, and expression profiles.[J]. Journal of Experimental Botany, 2009, 60(15):4383-4396.

Cambi A, Koopman M, Figdor C G. How C-type lectins detect pathogens[J]. Cellular microbiology, 2005, 7(4):481-488.

Cannon S B , Mitra A , Baumgarten A, et al. The roles of segmental and tandem gene duplication in the evolution of large gene families in Arabidopsis thaliana[J]. BMC Plant Biology, 2004, 4(1):10-0.

Cheng X, Wu Y, Guo J, et al. A rice lectin receptor-like kinase that is involved in innate immune responses also contributes to seed germination[J]. The Plant Journal, 2013, 76(4):687-698.

Christine Hervé, Jérome Serres, Dabos P, et al. Characterization of the Arabidopsis LecRK-a genes: members of a superfamily encoding putative receptors with an extracellular domain homologous to legume lectins[J]. Plant Molecular Biology, 1999, 39(4):671-682.

Deng K, Wang Q, Zeng J, et al. A Lectin Receptor Kinase Positively Regulates ABA Response during Seed Germination and Is Involved in Salt and Osmotic Stress Response[J]. Journal of Plant Biology, 2009, 52(6):493-500.

Desclos Theveniau M, Arnaud D, Huang T Y, et al. The Arabidopsis Lectin Receptor Kinase LecRK-V.5 Represses Stomatal Immunity Induced by Pseudomonas syringae pv. tomato DC3000[J]. PLoS Pathogens, 2012, 8(2):e1002513.

Edgar R C. MUSCLE: Multiple Sequence Alignment with High Accuracy and High Throughput[J]. Nucleic Acids Research, 2004, 32(5):1792-1797.

Finn R D , Penelope C , Eberhardt R Y , et al. The Pfam protein families database: towards a more sustainable future[J]. Nucleic Acids Research(D1):D1.

Gu Z, Cavalcanti A, Chen F C, et al. Extent of Gene Duplication in the Genomes of Drosophila, Nematode, and Yeast[J]. Molecular Biology and Evolution, 2002, 19(3):256-262.

Guo $C$, Yang X, Wang $Y$, et al. Identification and mapping of ts (tender spines), a gene involved in soft spine development in Cucumis sativus[J]. Theoretical and Applied Genetics, 2017. 
He X J, Zhang Z G, Yan D Q, et al. A salt-responsive receptor-like kinase gene regulated by the ethylene signaling pathway encodes a plasma membrane serine/threonine kinase[J]. Theoretical and Applied Genetics, 2004, 109(2):377-383.

Hofberger J A, Nsibo D L, Govers F, et al. A Complex Interplay of Tandem and Whole Genome Duplication Drives Expansion of the L-Type Lectin Receptor Kinase Gene Family in the Brassicaceae[J]. Genome Biology and Evolution, 2015, 7(3):720-734.

Hu B, Jin J, Guo A Y, et al. GSDS 2.0: An upgraded gene feature visualization server[J]. Bioinformatics, 2014, 31(8):1296.

Huang S W, Li R Q, Zhang Z H, et al. The genome of the cucumber, Cucumis sativus L[J]. Nature Genetics $2009,41,1275-1281$.

Jeffares D C, Penkett C J, Jürg Bähler. Rapidly regulated genes are intron poor[J]. Trends in Genetics, 2008, 24(8):375-378.

Johnson L S, Eddy S R, Portugaly E. Hidden Markov model speed heuristic and iterative HMM search procedure[J]. Bmc Bioinformatics, 2010, 11(1):431-0.

Kent W J, Baertsch R, Hinrichs A, et al. Evolution\"s cauldron: Duplication, deletion, and rearrangement in the mouse and human genomes[J]. Proceedings of the National Academy of Sciences, 2003, 100(20):11484-11489.

Ivica L, Peer B. 20 years of the SMART protein domain annotation resource[J]. Nucleic Acids Research (D1): D1.

Livak K, Schmittgen T. Analysis of Relative Gene Expression Data Using Real-Time Quantitative PCR and the $2^{-\triangle \Delta \mathrm{Ct}}$ Method[J]. Methods, 2000, 25 (4).

Li C H, Wang G, Zhao J L, et al. The Receptor-Like Kinase SIT1 Mediates Salt Sensitivity by Activating MAPK3/6 and Regulating Ethylene Homeostasis in Rice[J]. The Plant Cell, 2014, 26(6):2538-2553.

Liu P L , Huang Y, Shi P H, et al. Duplication and diversification of lectin receptor-like kinases (LecRLK) genes in soybean[J]. Scientific Reports, 2018, 8(1):5861.

Mehan M R, Freimer N B, Ophoff R A. A genome-wide survey of segmental duplications that mediate common human genetic variation of chromosomal architecture[J]. Human Genomics, 2004, 1(5):335--344 .

Ohtake Y. Salicylic Acid Induces the Expression of a Number of Receptor-Like Kinase Genes in Arabidopsis thaliana[J]. Plant and Cell Physiology, 2000, 41(9):1038-1044. 
Olds T, Mitchell M T, Koch M A, et al. Comparative evolutionary analysis of chalcone synthase and alcohol dehydrogenase loci in Arabidopsis, Arabis, and related genera (Brassicaceae)[J]. Molecular Biology \& Evolution, 2000, 17(10):1483.

Ranf S, Gisch N, SchaFfer M, et al. A lectin S-domain receptor kinase mediates lipopolysaccharide sensing in Arabidopsis thaliana[J]. Nature Immunology, 2015, 16(4):426-433.

Riou C, Christine Hervé, Valérie Pacquit, et al. Expression of an Arabidopsis lectin kinase receptor gene, lecRK-a1, is induced during senescence, wounding and in response to oligogalacturonic acids[J]. Plant Physiology and Biochemistry (Paris), 2002, 40(5):431-438.

Shen Y, Liu J, Geng H, et al. De novo assembly of a Chinese soybean genome[J]. Science China (Life Sciences), 2018, v.61(08):3-16.

Shiu S H . Plant receptor-like kinase gene family : diversity, function, and signaling[J]. Sci. STKE. 2001, 2001(113):re22.

Shumayla , Shailesh S , Pandey A K, et al. Molecular Characterization and Global Expression Analysis of Lectin Receptor Kinases in Bread Wheat (Triticum aestivum)[J]. PLOS ONE, 2016, 11(4):e0153925.

Sushma N, Thanat C, Ripoll D R, et al. Structural modules for receptor dimerization in the S-locus receptor kinase extracellular domain[J]. Proceedings of the National Academy of Sciences of the United States of America, 2007, 104(29):12211-12216.

Timothy Bailey C E. Fitting a Mixture Model By Expectation Maximization to Discover Motifs in Biopolymers[C]// 1994.

Vaid N, Pandey P K, Tuteja N. Genome- wide analysis of lectin receptor-like kinase family from Arabidopsis and rice[J]. Plant Molecular Biology, 2012, 80(4-5): 365-388.

Voorrips R E. MapChart: Software for the Graphical Presentation of Linkage Maps and QTLs[J]. Journal of Heredity, 2002, 93(1):77-78.

Wan J, Patel A, Mathieu M, et al. A lectin receptor-like kinase is required for pollen development in Arabidopsis[J]. Plant Molecular Biology, 2008, 67(5):469-482.

Wang Y , Cordewener J H G , America A H P , et al. Arabidopsis Lectin Receptor Kinases LecRK-IX.1 and LecRK-IX.2 Are Functional Analogs in Regulating Phytophthora Resistance and Plant Cell Death[J]. Molecular Plant-Microbe Interactions, 2015, 28(9):1032-1048.

Wang Y, Tang H, Debarry J D, et al. MCScanX: a toolkit for detection and evolutionary analysis of gene synteny and collinearity[J]. Nucleic Acids Research, 2012, 40(7):e49-e49. 
Wei G , Tian P , Zhang F , et al. Integrative Analyses of Non-targeted Volatile Profiling and Transcriptome Data Provide Molecular Insight into VOC Diversity in Cucumber Plants (Cucumis sativus L.)[J]. Plant Physiology, 2016, 172(1):603-618.

Wu $T$, Wang $R$, Xu X, et al. Cucumis sativus L-type lectin receptor kinase (CsLecRK) gene family response to Phytophthora melonis, Phytophthora capsici and water immersion in disease resistant and susceptible cucumber cultivars[J]. Gene, 2014, 549(2):214-222.

Yang S, Zhang X, Yue J X, et al. Recent duplications dominate NBS-encoding gene expansion in two woody species[J]. Molecular Genetics and Genomics, 2008, 280(3):187-198.

Yang Y, Labbé, Jessy, Muchero W, et al. Genome-wide analysis of lectin receptor-like kinases in Populus[J]. BMC Genomics, 2016, 17(1):699.

Yang Y, Labbé, Jessy, Muchero W, et al. Genome-wide analysis of lectin receptor-like kinases in Populus[J]. BMC Genomics, 2016, 17(1):699.

Zhang, Zhang, Xiao Q, et al. KaKs_Calculator: Calculating Ka and Ks through Model Selection and Model Averaging[J]. Genomics Proteomics Bioinformatics, 2006(4):259-263.

Zuo K, Zhao J, Wang J, et al. Molecular Cloning and Characterization of GhlecRK, a Novel Kinase Gene with Lectin-like Domain from Gossypium hirsutum[J]. DNA Sequence-The Journal of Sequencing and Mapping, 2004, 15(1):58-65.

\section{Table}

Table 1.

Basic character of CsLecRLK family 


\begin{tabular}{|c|c|c|c|c|c|c|c|c|c|c|c|}
\hline Group & Gene ID & Chr & Start & End & Strand & $\begin{array}{c}\text { Gene } \\
\text { (bp) }\end{array}$ & $\begin{array}{l}\text { CDS } \\
\text { (bp) }\end{array}$ & $\begin{array}{l}\text { Protein } \\
\text { (aa) }\end{array}$ & $\begin{array}{c}\mathrm{MW} \\
(\mathrm{KDa})\end{array}$ & pl & Localization \\
\hline \multirow{23}{*}{$\begin{array}{l}\text { C-type } \\
\text { L-type }\end{array}$} & Csa1G056960 & 1 & 6245947 & 6249211 & + & 3,265 & 1,674 & 557 & 62.5 & 9.51 & PM \\
\hline & Csa1G004050 & 1 & 615237 & 617749 & + & 2,513 & 1,977 & 658 & 73.8 & 5.23 & PM \\
\hline & Csa1G073890 & 1 & 7608750 & 7610584 & + & 1,835 & 1,794 & 597 & 66.5 & 6.14 & $\mathrm{CY}$ \\
\hline & Csa2G439150 & 2 & 22949410 & 22951362 & + & 1,953 & 1,953 & 650 & 72.6 & 6.84 & PM \\
\hline & Csa2G439210 & 2 & 22985725 & 22987680 & + & 1956 & 1956 & 651 & 72.2 & 6.73 & PM \\
\hline & Csa3G048440 & 3 & 3434259 & 3436274 & - & 2,016 & 2,016 & 671 & 73.8 & 7.39 & PM \\
\hline & Csa3G115060 & 3 & 6101289 & 6103476 & - & 2,188 & 2,058 & 685 & 76.5 & 6.35 & PM \\
\hline & Csa3G115090 & 3 & 6119460 & 6122293 & - & 2,834 & 1,908 & 635 & 71.9 & 6.66 & PM \\
\hline & Csa3G730910 & 3 & 27403916 & 27405718 & + & 1,803 & 1,803 & 600 & 66.4 & 5.25 & PM \\
\hline & Csa3G730920 & 3 & 27408398 & 27410741 & + & 2,344 & 2,044 & 667 & 74.2 & 7.04 & PM \\
\hline & Csa3G734030 & 3 & 27978179 & 27980785 & + & 2,607 & 2,052 & 683 & 75.1 & 7.41 & PM \\
\hline & Csa3G734040 & 3 & 27981843 & 27984009 & + & 2,167 & 1,986 & 661 & 73.3 & 5.62 & PM \\
\hline & Csa3G734050 & 3 & 27985087 & 27987243 & + & 2,157 & 2,121 & 706 & 78.4 & 8.13 & PM \\
\hline & Csa3G736960 & 3 & 28496446 & 28499101 & - & 2,656 & 2,184 & 727 & 78.5 & 5.62 & PM \\
\hline & Csa4G296230 & 4 & 12033379 & 12039859 & - & 6,481 & 2,004 & 667 & 75.3 & 7.41 & PM \\
\hline & Csa4G296250 & 4 & 12055822 & 12057932 & + & 2,111 & 1,980 & 659 & 73.5 & 6.71 & PM \\
\hline & Csa5G648630 & 5 & 27485882 & 27487867 & + & 1,986 & 1,986 & 661 & 73.3 & 6.68 & PM \\
\hline & Csa6G338050 & 6 & 15414546 & 15416705 & + & 2,160 & 2,088 & 695 & 76.9 & 6.45 & PM \\
\hline & Csa7G029930 & 7 & 1577321 & 1580540 & + & 3,220 & 2,076 & 691 & 78.1 & 6.55 & CY \\
\hline & Csa7G048050 & 7 & 2990460 & 2992838 & + & 2,379 & 2,058 & 685 & 76.2 & 5.99 & PM \\
\hline & Csa7G067400 & 7 & 4115966 & 4118216 & - & 2,251 & 2,133 & 710 & 79.5 & 6.82 & PM \\
\hline & Csa7G067410 & 7 & 4119365 & 4121401 & - & 2,037 & 2,037 & 678 & 75.4 & 6.04 & PM \\
\hline & Csa7G067430 & 7 & 4124769 & 4127087 & + & 2,319 & 2,124 & 707 & 79.7 & 6.3 & PM \\
\hline \multirow[t]{23}{*}{ G-type } & Csa1G071150 & 1 & 7119234 & 7121709 & + & 2,476 & 1,773 & 590 & 66.4 & 5.18 & PM \\
\hline & Csa1G071160 & 1 & 7124699 & 7127292 & - & 2,594 & 1,818 & 605 & 68.1 & 7.46 & PM \\
\hline & Csa1G071170 & 1 & 7134918 & 7137465 & - & 2,548 & 1,746 & 581 & 65.6 & 7.26 & PM \\
\hline & Csa1G071270 & 1 & 7179289 & 7181813 & + & 2,525 & 1,935 & 644 & 73.1 & 6.98 & PM \\
\hline & Csa1G605730 & 1 & 23772913 & 23775291 & - & 2,379 & 2,379 & 792 & 89.1 & 6.24 & PM \\
\hline & Csa1G605740 & 1 & 23777925 & 23780300 & - & 2,376 & 2,376 & 791 & 89.4 & 5.46 & PM \\
\hline & Csa1G605750 & 1 & 23782466 & 23785387 & - & 2,922 & 2,439 & 812 & 91.1 & 5.28 & PM \\
\hline & Csa3G099580 & 3 & 4888747 & 4892235 & - & 3,489 & 2,238 & 745 & 84.7 & 8.97 & PM \\
\hline & Csa3G733860 & 3 & 27894955 & 27899854 & - & 4,900 & 2,502 & 833 & 94.5 & 6.32 & PM \\
\hline & Csa3G733880 & 3 & 27905835 & 27908289 & - & 2,455 & 2,421 & 806 & 90.5 & 6.22 & PM \\
\hline & Csa4G005510 & 4 & 865713 & 869014 & - & 3,302 & 2,460 & 819 & 92.1 & 6.81 & PM \\
\hline & Csa4G288620 & 4 & 11220419 & 11222863 & - & 2445 & 2445 & 736 & 82.6 & 5.76 & PM \\
\hline & Csa4G289620 & 4 & 11230649 & 11233131 & - & 2,483 & 2,358 & 785 & 88.6 & 7.69 & PM \\
\hline & Csa4G289630 & 4 & 11233952 & 11236384 & - & 2,433 & 2,184 & 727 & 81.9 & 4.98 & PM \\
\hline & Csa4G289640 & 4 & 11237240 & 11239680 & - & 2,441 & 2,424 & 807 & 90.7 & 5.84 & PM \\
\hline & Csa4G289650 & 4 & 11240557 & 11242883 & - & 2,327 & 2,136 & 711 & 79.4 & 5.64 & PM \\
\hline & Csa4G290150 & 4 & 11244840 & 11247660 & - & 2,821 & 2,094 & 697 & 77.9 & 7.25 & PM \\
\hline & Csa5G550210 & 5 & 19486884 & 19489396 & + & 2,513 & 1,887 & 628 & 70.3 & 8.89 & PM \\
\hline & Csa5G550230 & 5 & 19498199 & 19500774 & - & 2,576 & 2,478 & 825 & 92.3 & 6.66 & PM \\
\hline & Csa6G052130 & 6 & 3992021 & 3994920 & + & 2,900 & 2,196 & 731 & 79.6 & 5.48 & Ec \\
\hline & Csa6G516770 & 6 & 26894261 & 26897704 & - & 3,444 & 2,142 & 713 & 79.8 & 5.91 & PM \\
\hline & Csa7G045520 & 7 & 2678627 & 2681841 & - & 3,215 & 2,496 & 831 & 93.9 & 5.37 & Ec \\
\hline & Csa7G446780 & 7 & 17724360 & 17728540 & - & 4,181 & 2,421 & 806 & 91.9 & 5.53 & PM \\
\hline Averger & & & & & & 2,674 & 2,117 & 703 & 79 & 7 & \\
\hline
\end{tabular}

Abbreviations: $C y$ cytosol, $E c$ extracellular, $P M$ plasma membrane, $C D S$ coding sequence 


\section{Supplementary Information}

Additional file 1: Figure S1. Motif Structure. (PDF 450 kb)

Additional file 2: Table S1. Promoter elements analysis of 46 CsLecRLK genes. (XLSX 303 kb)

Additional file 3: Table S2. RNA-Seq data of 46 CsLecRLK genes in different tissue. (XLSX 15kb)

Additional file 4: Figure S2. Promoter Analysis of CsLecRLKs (PDF 2,382 kb)

Different color and shape represent different cis-acting elements.

Additional file 5: Table S3. Primers for qRT-PCR in this study. (PDF 130 kb)

Additional file 6: Table S4. CDS sequences of C-type CsLecRLKs. (PDF 89 kb)

Additional file 7: Table S5. Protein sequences of C-type CsLecRLKs. (PDF 90 kb)

Additional file 8: Table S6. CDS sequence of G-type CsLecRLKs. (PDF 137 kb)

Additional file 9: Table S7. Protein sequences of G-type CsLecRLKs. (PDF 62 kb)

Additional file 10: Table S8. CDS sequences of L-type CsLecRLKs. (PDF 140 kb)

Additional file 11: Table S9. Protein sequences of L-type CsLecRLKs. (PDF 54 kb)

Figures 
A

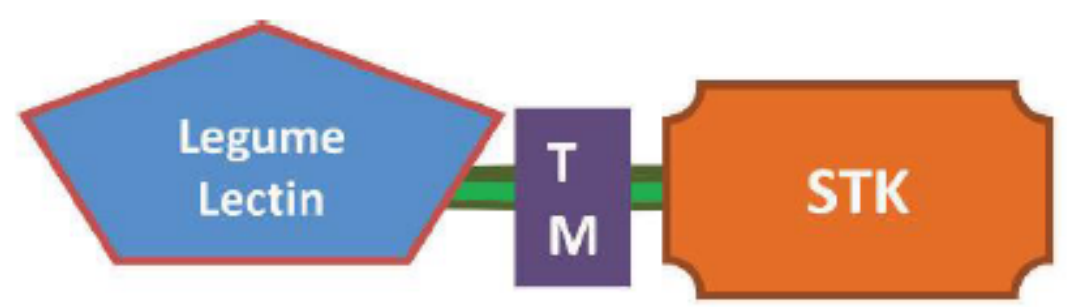

B

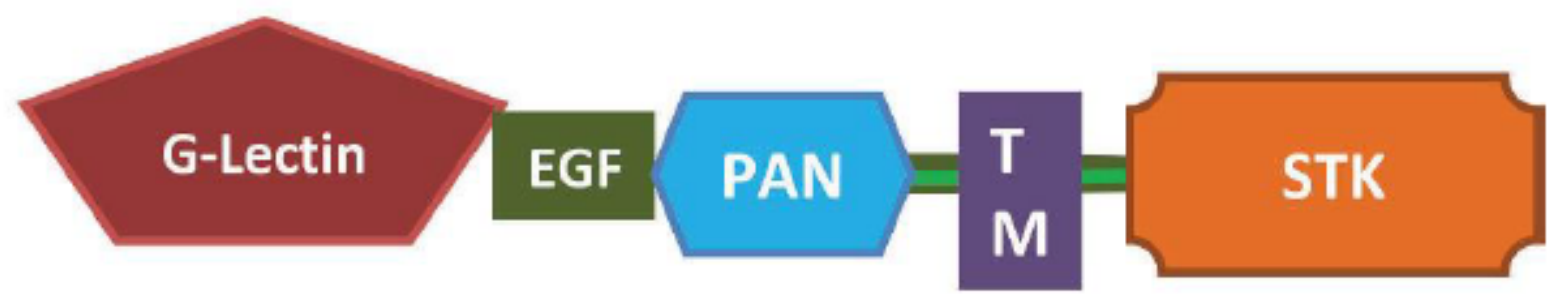

C

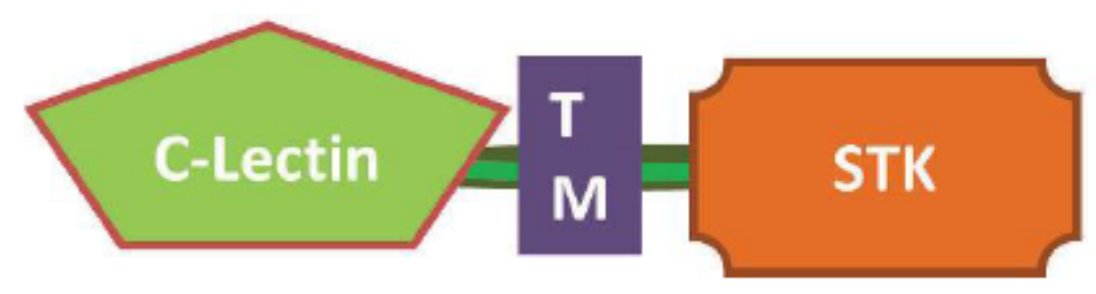

Figure 1

Line Model of the Three LecRLK Classes (Vaid et al. 2013). On the basis of extracellular domain, the LecRLKs are classified into three classes: L-type, G-type, and C-type. (A) L-type LecRLK with legume lectinlike extracellular domain, (B) G-type LecRLK with a-mannose binding bulb lectin domain. This class of proteins also possesses a PAN and/or an EGF domain, (C) C-type LecRLKs characterized by the presence of calcium-dependent carbohydrate-binding domains. TM: transmembrane region, STK: a cytoplasmic Serine/Threonine kinase domain. 


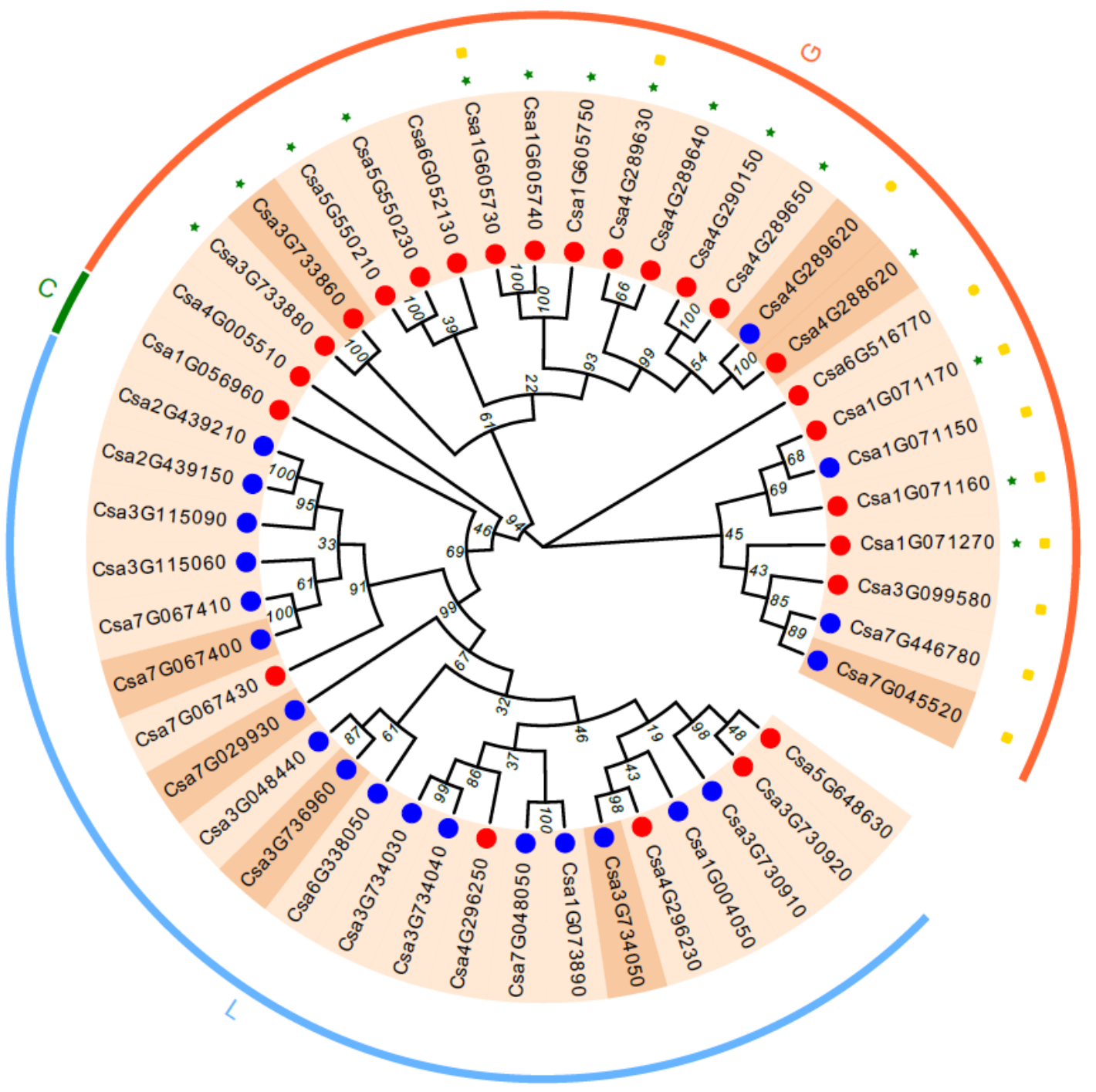

Figure 2

Unrooted Phylogenetic tree construction and structure analysis of CsLecRLKs The $L, G$ and $C$ respectively represented different L-type, G-type and C-type LecRLK subfamilies. The blue circles represented proteins with a signal peptide, the red circles proteins without a signal peptide. The green stars represented proteins contained EGF domains, the yellow squares represented proteins contained PAN domains. The sandybrown leaves represented proteins only contained one transmembrane domain, the peachpuff leaves represented proteins containing more than one transmembrane domain. 


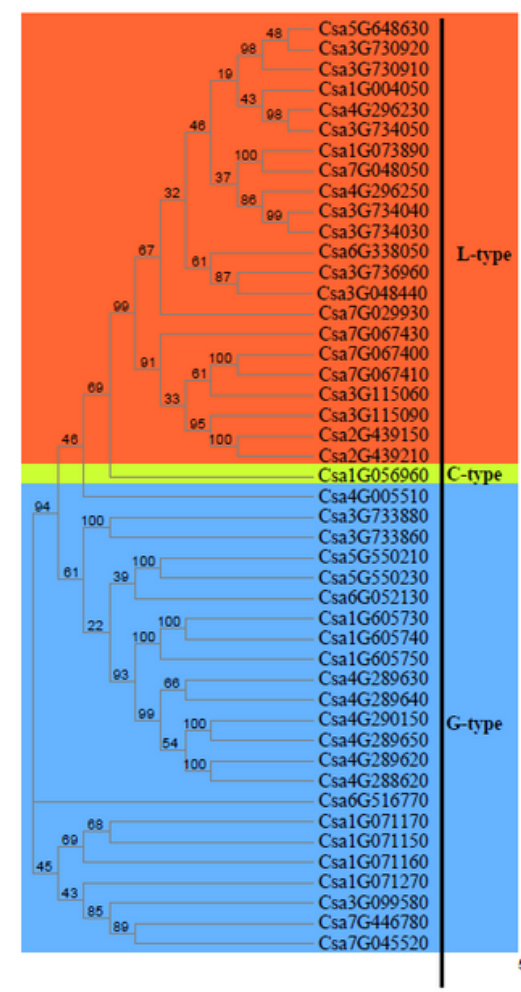

A

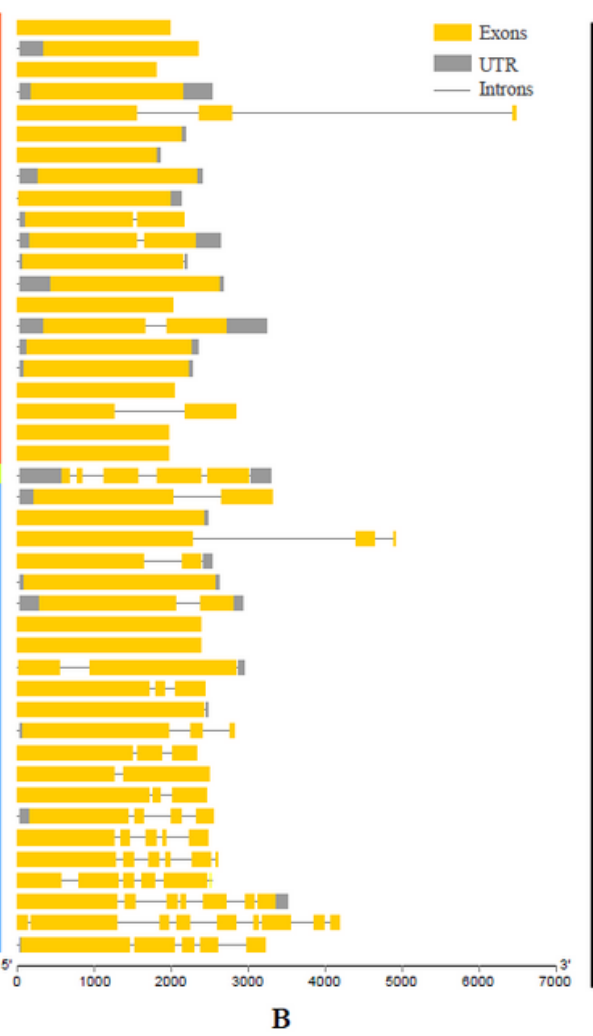

B

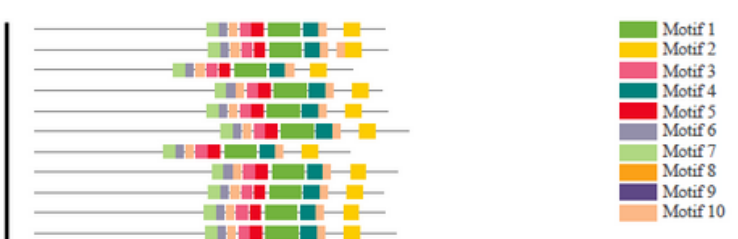

Figure 3

An analytical view of the CsLecRLK genes The following parts are shown from left to right. (A) Protein maximum likelihood tree: The tree was constructed using a maximum-likelihood method, and bootstrap values were calculated with 1,000 replications using MEGA 7.0.21. (B) Gene structure: The lines represented the intron. The grap square represented the $3^{\prime}$ UTR and the 5' UTR. The yellow square represented the exon. (C) Protein structure: The search for 10 common motifs shared among the CsLecRLK proteins, different color represented different motifs.

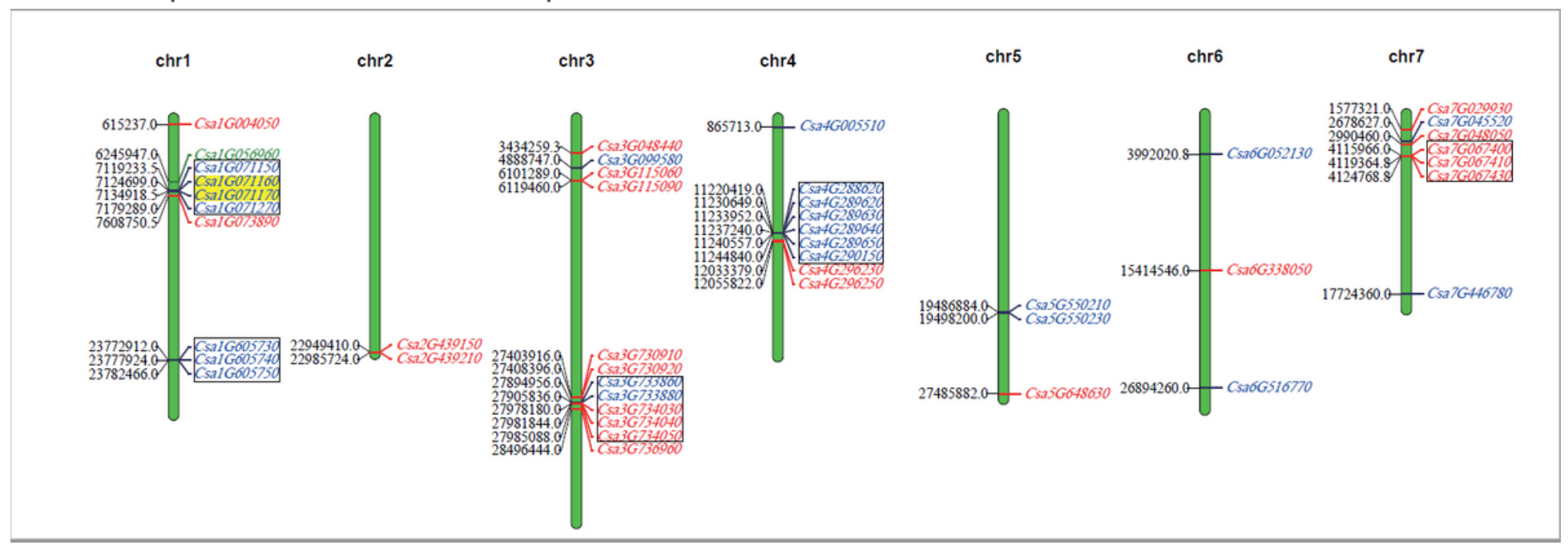


Chromosomal distribution and gene duplications of CsLecRLK genes The number at the chromosome (left) represented the position of CsLecRLK genes, and the right information on the chromosome (right) represented the gene ID of CsLecRLKs. The tandem duplicated genes are represented by yellow background, and the gene clusters are boxed together by black lines. The red represented L-type CsLecRLKs, the green represented C-type CsLecRLKs, the blue represented G-type CsLecRLKs.

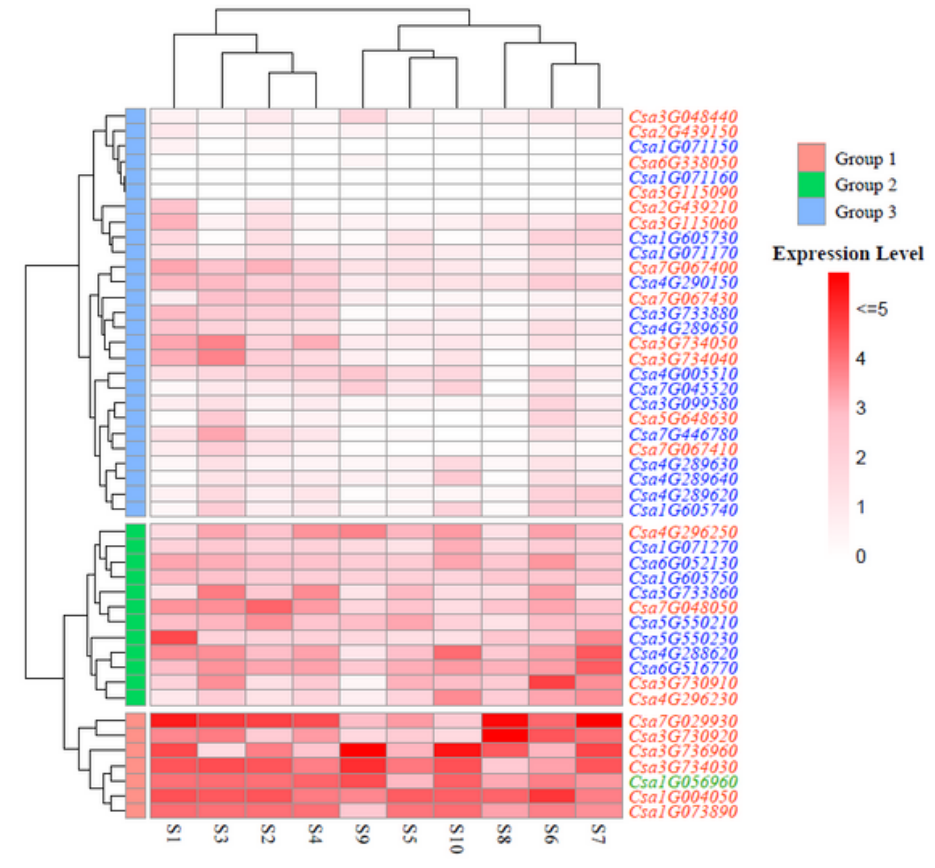

A

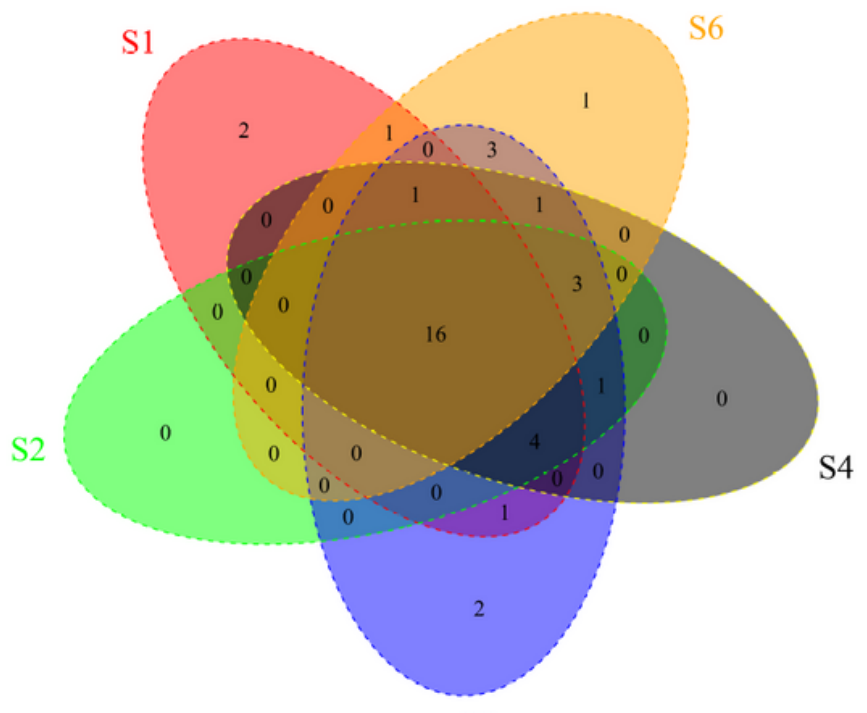

S3

\section{Figure 5}

Expression profile of CsLecRLK gene family in different tissues (A) Heatmap depicting the expression profile of CsLecRLK family in different developmental tissues. The red represents L-type CsLecRLKs, the green represents C-type CsLecRLKs, the blue represents G-type CsLecRLKs; (B) Venn diagram depicting the distribution of shared expression of CsLecRLKs under 5 typical tissues. The following abbreviations are used: S1, roots of 4-week-old seedlings; S2, hypocotyl of 4-week-old seedlings; S3, cotyledon of 4week-old seedlings; S4, true leaf of 4-week-old; S5, Stem; S6, Tendril; S7, female flower; S8, male flower; S9, unfertilized ovary; $\mathbf{S 1 0}$, peel of unfertilized ovary. 


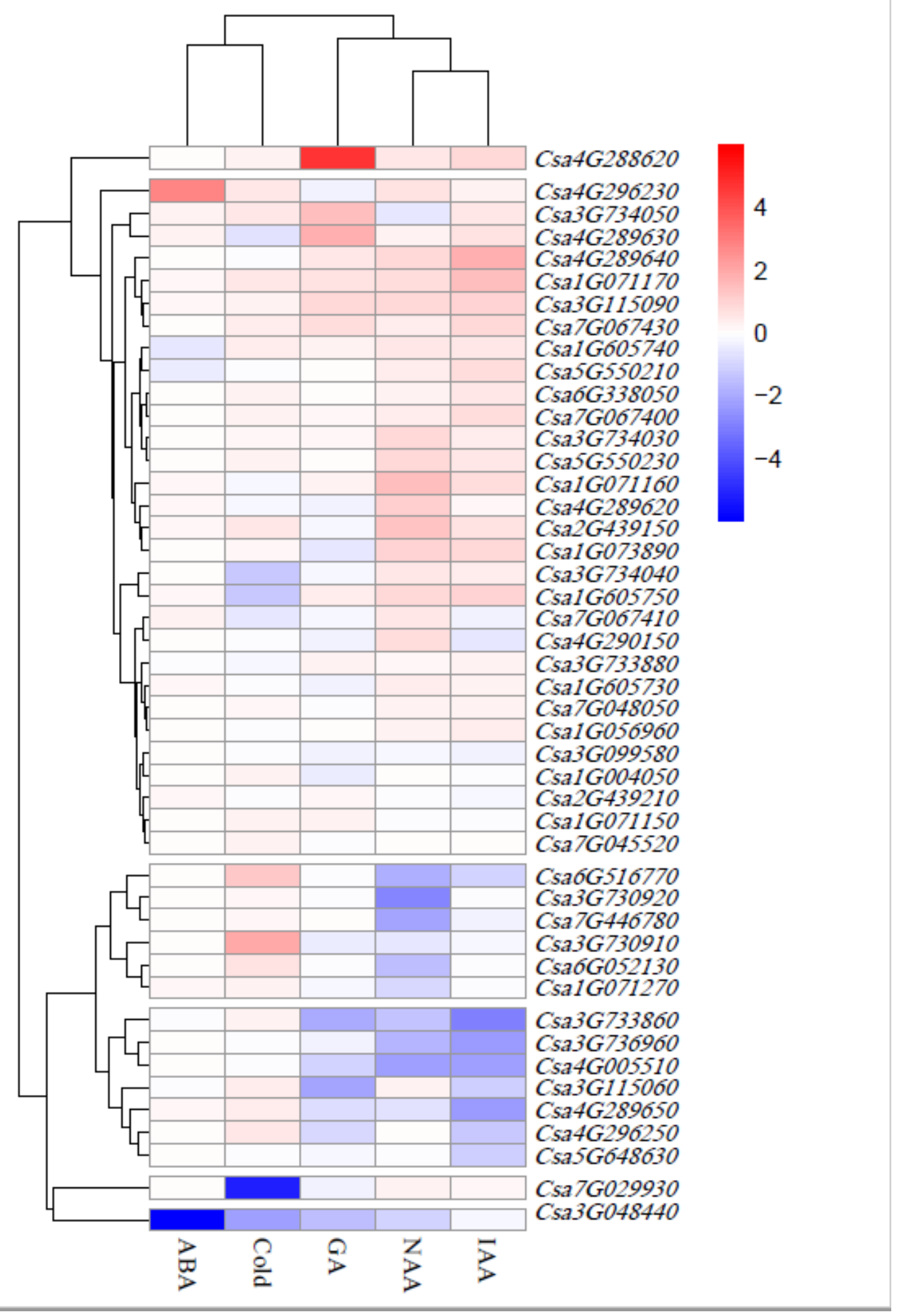

Figure 6

Expression analysis of CsLecRLK genes in response to different treatments The Heatmap represented the fold change of expression level under 5 treatments, GA, ABA, IAA, NAA and cold stress.

\section{Supplementary Files}


This is a list of supplementary files associated with this preprint. Click to download.

- Additionalfile6Tables4.pdf

- MotifStracture.pdf

- Ctypeprotein.pdf

- Gtypeprotein.pdf

- GtypeCDS.pdf

- CtypeCDS.pdf

- Additionalfile3Tables2.xlsx

- LtypeCDS.pdf

- Ltypeprotein.pdf

- Additionalfile4Figures2.pdf

- Additionalfile2TableS1.xlsx

- Additionalfile10TableS8.pdf

- Additionalfile11Tables9.pdf

- Additionalfile8Tables6.pdf

- Additionalfile9Tables7.pdf

- Additionalfile7Tables5.pdf

- Additionalfile1Figures1.pdf

- Additionalfile5Tables3.pdf 